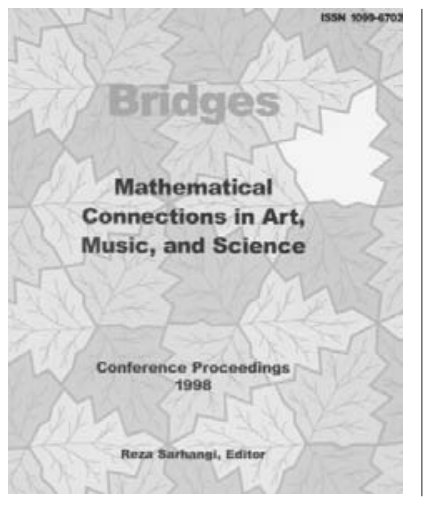

Reza Sarhangi ed.

Bridges: Mathematical Connections in Art, Music, and Science.

Conference Proceedings 1998.

Winfield, Kansas: Southwestern College, 1998

Reviewed by Solomon Marcus.

\title{
The Emergence of Bridges
}

The key word, giving the motivation of the most contributions in this volume, is BRIDGES. The title of the volume is just the slogan under which the Conference was announced at the beginning of the year 1998. This slogan requires some explanations. The noun "bridge" and the verb "to bridge" became, in the last decades, one of the most frequent metaphors to which a reference is made when the adopted approach crosses different fields. Is it equivalent to what is traditionally called interdisciplinarity? It seems that "bridge" is in some sense less and in some other sense more. We will not speak of bridging mathematics and mechanics, because their collaboration is very old. "Bridge" is reserved for fields or trends not yet connected or insufficiently connected. But this is not the only difference. Interdisciplinarity is usually understood in a unidirectional binary way: some ideas, tools, results or methods from a discipline A are used in approaching a problem in another discipline B. "Bridging A and B" may refer also to the case when $\mathrm{A}$ and $\mathrm{B}$ are entities of another type than disciplines, for instance one can bridge nature and culture (the slogan of an international congress of semiotics was "Semiotics Bridging Nature and Culture"), the quantum level and the cosmic level of reality (like in quantum cosmology), exactness and abstraction (like in mathematics), Plato and Leonardo da Vinci (like in some artistic works), objective and subjective (like in some philosophical approaches), etc. Moreover, "bridging A and B" may refer to a bidirectional action, and this is exactly what happens between art and mathematics. For instance, the "clapping music" proposed by Steve Reich (see Joel K. Haack's contribution in this volume) can be better understood by means of mathematics, but, in its turn, this music is a source of new mathematical problems, so both music and mathematics take some profit in this interaction. Besides this, bridging does not refer only to two entities, it may function among several entities. For instance, when we say that Mandelbrot's fractal geometry bridges mathematics, meteorology, turbulence, population ecology, and economics, we mean that fractal objects occur in all these domains, so fractalness appears as a common denominator of some very heterogeneous fields. The contributions in this volume are rich in all kinds of bridges; but only 
some of them are explicit, many of them remain implicit, only suggested. For instance, reading Golubitski \& Melbourne's article (p. 210), we learn from the beginning that mathematics will be used to solve a problem concerning the classification of architectural columns; but at the end of this article it is said, "Our discussion on symmetry and columns was a direct consequence of our participation in an interdisciplinary seminar on 'The Biology of Beauty'. So, we realize that biology was also involved, at least indirectly, in the proposed approach.

\section{Transdisciplinarity and Universal Paradigms}

There is now a new term proposed to cover approaches going across the existing disciplines, among them and beyond them: transdisciplinarity. The difference from interdisciplinarity is in several respects. First of all, transdisciplinarity is coping with problems that cannot be formulated (and a fortiori solved) in terms of only one discipline. For instance, when we look for a common pattern of the biological, the psychological, and the social time, we are placed from the beginning in a transdisciplinary perspective. Then, transdisciplinarity may ignore the existence of disciplines and try to realize a fresh look at the world, independent of any preexisting entities. In this respect, transdisciplinarity goes further than the bridging approach, but any bridge is a part of a transdisciplinarity approach. This is the reason we will pay attention in this section to transdisciplinarity, in order to check its possible higher explanatory capacity in respect to art, math, and other fields, such as religion and philosophy. In 1993, the International Center of Transdisciplinary Research and Studies was founded in Paris. Its first world congress took place at Convento da Arrabida, Portugal, November 1994, and adopted the "Charter of Transdisciplinarity", whose Article 5 says, "The transdisciplinary vision is determinedly open in that it transcends the field of exact sciences by encouraging them to communicate and to be reconciled with not only the humanities and the social sciences, but also with art, literature, poetry and spiritual experience". (See, for more, Basarab Nicolescu, La Transdisciplinarite, Editions du Rocher, Paris, 1996.) While transdisciplinarity, in this French version, is strongly based on the ideas of quantum mechanics, claiming the existence of different levels of reality, for instance, the quantum level and the macroscopic level, ruled by completely different laws and different logics, other versions of transdisciplinarity were also proposed. Let us quote in this respect Pierre Weil, Ubirata D'Ambrosio, and Roberto Crema, Rumo a nova transdisciplinaridade, Summus Editorial, Sao Paulo, 1993, where transdisciplinarity is mainly understood in the integrative, holistic view promoted by the information-computation era. It may be interesting to observe that Brazil and some EasternEuropean countries, with a great delay in their cultural development, became more open in bridging art and science, just in view of the absence of a different long cultural tradition that could be an obstacle to the promotion of new ideas. The whole trend of modern art, convergent at the beginning of the XXth century with the new geometries and the new physics, got great support from some artists and writers coming from Eastern-Europe (the dadaism was founded by the Romanian Tristan Tzara). One of the most important painters of modern Brazil, Samson Flexor, bridging his art with modern science, was born in Eastern Europe before the first world war. He became a French modernist, but only in Brazil does he reach his highest performance.

A special type of transdisciplinarity is realized by means of the so-called universal paradigms, 
such as sign, time, symmetry, complexity, information, computation, paradox, imprecision, etc. As a matter of fact, the bridge can be also considered such a universal paradigm. The universality refers here to the fact the respective entities are involved in the quasi-totality of natural and social processes. Each of these paradigms (the word is used here in the sense of Thomas Kuhn's The Structure of Scientific Revolutions) leads to a segmentation of knowledge other than the segmentation in disciplines, as it was essentially proposed by Auguste Comte, in the first half of the XIXth century. This means that each such segmentation goes across the disciplines and may go beyond them, just as in the spirit of transdisciplinarity. Particularly, each universal paradigm leads to a specific way to bridge art and science.

\section{The Crisis of Bibliographic Information}

The situation described above shows the difficulty in being systematically informed in the huge bibliography related to these non-classical topics. For mathematics, physics, chemistry, biology we have an international system of review journals and of citation. For the transdisciplinary topics the available means of bibliographic orientation are very poor and to a large extent random. Bridging, transdisciplinarity and universal paradigms are to a large extent still a no-man's-land. In the present volume too, like in most writings of this nature, the same phenomenon can be observed. Excepting some periodic sources such as the journals Leonardo and Computers and the Humanities, most bibliographic sources related to art and science are spread in a huge variety of books and journals nobody can keep under observation. It is enough to look in the section "References" of the articles in this volume to realize this diversity, but also the great gaps existing in this respect. Let us refer to the article by Kim Williams, a remarkable one, pointing out one of the most advanced ideas related to mathematics as a catalyst in relating architecture to other fields, whether they are arts or sciences. As a matter of fact, mathematics has long been a catalyst of a great number of transfers of ideas or results from one field to another. (An example: the transfer of the concept of entropy from thermodynamics to information theory and from the latter to linguistics, literature, and art - see Arnheim - became possible due to the mathematical (logarithmic) expression of this concept). In this respect, Williams makes reference to Mario Salvadori, whose work is entitled, "Can There be any Relationships Between Mathematics and Architecture?", which was published in 1996, in a collective volume having Williams as editor. It seems very strange to formulate such a doubt in 1996, after an itinerary of several decades of beneficial interaction of the respective fields (leaving aside the acquisitions of Greek antiquity and of Renaissance as well as of some further developments). Fields such as pattern recognition and picture grammars, the collective volumes dedicated to symmetry (those published by the International Society for Symmetric Studies and those edited by Professor Istvàn Hargittai) as well as those dedicated to the semiotics of architecture, give many references in this respect. In 1982, I coordinated the collective volume, The Mathematical Semiotics of Visual Arts (in Romanian, Scientific and Encyclopedic Publishing House, Bucharest), where the mathematics of semiotics has its place, mainly via formal grammars and picture grammars. The bibliography of this topic was already very rich at that time, and the efficiency of this bridge for architecture was beyond any doubt. Sometimes, the mathematics 
of architecture comes in a very indirect way, and the example I will give shows how mathematics can create a bridge between architecture, linguistics, and molecular genetics. When I attended the International Summer School in Semiotics (Urbino, Italy) in 1973, I gave a lecture about the use of mathematical linguistics in molecular genetics. One of the participants, Martin Krampen, a leader in the semiotics of architecture, told me after the lecture that the model I proposed is valid, mutatis mutandis, for architectural planning too. One year later, he included my model in his report on the semiotics of architecture, delivered at the First Congress of the International Association for Semiotic Studies (Milano, 1974). (See the respective proceedings, A Semiotic Landscape, S. Chatman, U. Eco and J. M. Klinkenberg, eds., Mouton, The Hague, 1979.) So, we are now able to make explicit what is still missing with our bridges. Mature science, mature research are like what is called in sport a relay race. We start from the point where the researchers before us arrived, taking their results, and trying to go further. The bibliographic references of our works are a symptom of this behavior, and the huge electronic work called "Science Citation Index" accounts for this practically infinite tree represented by the cognitive adventure. It happens, however, that this relay race type of research is still very weak in the field of art-science interaction and generally in the transdisciplinary trend of research. The other type of research, very frequent in this respect, is to tell a personal experience, with little care for possible interferences with other personal experiences or with some more systematic research on a similar topic. Brent Collins (p. 21-28) is telling us such a personal interesting reflection that cannot be ignored. The opposite case, of a research starting from a bibliography of the problem, is that of Reza Sarhangi and Bruce D.Martin (p. 93-111), giving us the feeling that the authors tried, and to a large extent succeeded, to cover as much as possible from two thousands of reflection about circularity. Most contributions, however, are somewhere between these two extremes.

\section{Strange, Misleading, Missing}

The word "art" is used in various acceptions. Obviously, for the editor of these Proceedings, Professor Reza Sarhangi, who was also the organizer of the Conference, "art" means, like for many other people, "visual art". However, in some parts of the world, like the part where I am living, art includes music, too and sometimes, it also includes poetry and literature in general. Putting music beside art seems strange to me, in the same way in which, unfortunately, we frequently meet the syntagm "culture and science", suggesting that science does not belong to culture. Similarly, "mathematical connections in science" may suggest that mathematics is not a science. So, the slogan of these Proceedings may become misleading. As a matter of fact, according to the belief of many great mathematicians, mathematics is both art and science (see Armand Borel's book, Mathematik: Kunst und Wissenschaft), and this is true for other sciences too. For theoretical physics, the article of presentation in the French Encyclopaedia Universalis (vol. 14, 1968, p. 764-766) is entitled: "La poetique de la physique mathematique et l'ontologie fondamentale". The status of art, for chemistry, is splendidly argued by the Nobel laureat Roald Hoffmann (The Same and Not the Same, Princeton University Press, 1995). One can understand that the attitude prevailing in the bureaucracy of culture (systems of education, organization of research, ministries, etc.) is still dominated by the old atomistic, disciplinary vision of culture 
seen as a collection of boxes whose interaction is, to a large extent, ignored. After the long period of synchretism which dominated the history of culture until the Renaissance, a new period began, of increasing separation between science and the humanities. Well known in this respect is Blaise Pascal's dichotomy "esprit geometrique-esprit de finesse" calling attention, in the XVIIth century, to the irreducible opposition between two types of thinking, one prevailing in science, the other in the humanities. C. P. Snows's "two cultures", at the middle of the XXth century, is a modern version of Pascal's dichotomy. "Mathematical Connections in Art, Music, and Science" is a challenge to both Pascal and Snow. But what does "connections" mean? It may mean only that mathematics is involved in art, and this fact deserves attention. But how essential is this involvement? Does it concern the kernel of the artistic creativity or only its external part? Or maybe only the way to interpret it? Does mathematics change itself, does it become richer, when it is involved in artistic processes? All these questions have long been a challenge for culture and perhaps this challenge will remain forever. But one thing is sure; we will never have a chance to realize an essential involvement of mathematics in art, if we don't realize first, or at the same time, an artistic understanding of what mathematics is in its very nature. Connecting mathematics in a deep way to painting and to music is possible only if we discover concomitantly the artistic kernel of mathematics. In the light of this principle, we have to judge the contributions in this volume too.

One more problem: the title of this book ignores (deliberately?) the important interaction between mathematics and poetry, between mathematics and literature in general. As a consequence, the presence of literature in this book is very poor. Obviously, one can limit the topic under examination; but I don't think we can acquire a satisfactory understanding of the mathematics-art interaction if we exclude systematically some parts of the art. I was personally involved for several decades in the mathematics of poetry, and I know how rich and interesting the literature is in this field. This gap should be bridged in a subsequent edition of the Conferences of this type.

\section{Reasons to connect mathematics to art}

Most authors in Bridges are either artists (including art historians and educators) or mathematicians (including computer-scientists). Interesting to note: With respect to the total number of 37 contributions, only seven have more than one author and only five of them have authors from different fields. Topics could be classified, roughly speaking, in: visual arts, music, mathematics, semiotics, and poetry. Obviously, we have in view the main stress, because otherwise each contribution refers to a bridge requiring at least two terms.

Since the main actors of Bridges are mathematics and art, we propose five modalities in which the former can be involved in the latter: a) because you cannot avoid it; b) because it helps to improve the artistic creativity or at least its understanding; c) because it is useful, although not essentially involved; d) because, like some drugs, it does not damage; e) for snobbery, but at the expense of intelligibility and sometimes with the aim to hide the absence of ideas. There is no general method to locate a research in one or another of these categories; moreover, the border between them is fuzzy, and we can be in error very easy. Fortunately, no contribution in Bridges is in the category e, and perhaps category d, too, is not represented. 
In exchange, many contributions join the categories a and $\mathrm{b}$. The degree of involvement of mathematics in art should not be confused with the quantity of technical tools displayed. The right criterion is the explanatory capacity of some mathematical ideas, theorems, models, theories. But the quality of the mathematical involvement cannot guarantee the artistic value of a work; generally speaking, the former is neither necessary nor sufficient for the latter. In respect to the degree of originality, we are faced with the difficulties already pointed out concerning the lack of a systematic bibliographic information related to transdisciplinarity.

\section{Visual Arts}

Many contributions in this field join the category a. This happens because the whole evolution of visual arts was strongly related to the evolution of mathematics. Perspective, discovered during Renaissance, was the artistic source of development of a new chapter of geometry, projective geometry (with descriptive geometry associated to it). The history of architecture was so strongly related to mathematics that Mario Salvadori writes, "The relationships between mathematics and architecture are so many and so important that, if mathematics had not been invented, architects would have had to invent it themselves" (see the quotation given by Kim Williams, p. 17). Ironically, this reflection is inserted in an article whose title throws a doubt on the existence of the respective relationships; clearly, this doubt was purely rhethorical. Many contributions, if not all, start with the implicit presupposition that artistic beauty follows some order and simplicity, harmony and symmetry. Since these factors are guiding mathematics too, the general strategy is to use or to identify in art some mathematical regularities. Euclidean geometry and group theory are two preferred fields in this respect. For instance, Helena Verrill (p. 55) uses piecewise linear isometries of the plane, which are invariant under some crystallographic groups. Carlo H. Séquin (p. 1-10) stresses the technological aspect. $\mathrm{He}$ assumes that forms resulting from some optimization processes satisfy the requirements of artistic beauty. The virtual structures obtained by his generator program and the possibilities of their physical realization open, as he says, "a new artistic universe", but it is too early to understand their artistic status. Are Euclid (geometry), Galois (groups) and Felix Klein (invariants of some transformation groups) the only possible aesthetic ideal? Maybe, if we remain at the kind of beauty in great appreciation during the Greek antiquity and the Renaissance. However, already in the XIXth century new aesthetic trends emerged. One hundred years ago, the world of art began a big metamorphosis, under the influence of the new geometries and the new physics. The divorce from classical geometric and physical laws began its itinerary and we observe how most contributions in Bridges are influenced by this trend. One of the variants of this non-classical trend is related to the fractal geometry of nature, but a great misunderstanding persists here, due to the confusion between mathematical fractals and fractals in nature, the former being an idealized model of the latter. Mathematical fractals are invisible, so they are visually neither beautiful nor ugly. What is considered the visual side of fractals as beautiful forms is related to the mathematical approximation, usually by computers, of the mathematical fractals. Clouds, coasts are natural fractals, and they can be seen, but not understood; mathematical fractals, such as Koch curves, can be understood but not seen. No human hand can trace the graph of a curve everywhere devoid of tangent. Fractals are related to 
complexity and, some times, to ugliness; Euclidean geometry is related to simplicity and classical beauty. However, in the XIXth century the poetry of the ugliness became a new artistic program (see Baudelaire, in France). Fractals correspond to a new age of art.

Now, passionate about the apparently unlimited combinatorial and syntactic capacity of computers, we can no longer keep under control the respective semantics. This gap between syntax and meaning may lead to a kind of schizophrenia already observed by Errett Bishop (the famous author of Foundations of Constructive Analysis, McGraw Hill, New York, 1967) in a lecture given in 1973 at the American Mathematical Society entitled "The Schizophrenia of Contemporary Mathematics". This warning may suggest a possible schizophrenia of contemporary art, where schizophrenic mathematics and computer science are used. However, this danger should not be used as a global argument against the mathematical/information/ computation challenge in contemporary art. Any big positive change has some secondary negative effects. In order to prevent their amplification, we should not separate modern technology from the science behind it, and this science, in its turn, should not be separated from its cultural, empirical and philosophical roots. This imperative is equally valid for Séquin's already discussed contribution and for Gary R. Greenfield's research providing a model that both simplifies and refines Karl Sinus' art-by-choice technique known as "evolving expressions". We are in the field of biologically inspired computer art techniques. It is a long tradition to consider that art is inspired by the evolution of forms in the living universe. Now, with the first signs of a possible "biological computation" (see Adleman's pioneering experiment) and with the fast development of computational biology (stimulated by the huge Human Genome Project), we may expect much more from the artistic impact of the biology-computer science interaction. But by computer science we don't mean only its technological side, most visible, but not most essential; we mean primarily the whole mathematics, the whole logic, the tentative cognitive models, algorithms and programs which make possible the computation. All these things should be made more explicit, in order to make possible the understanding of the human intellectual effort and the cultural horizon of what is called so improperly, computer art. Let us recall Dijkstra's observation that calling informatics "computer science" is like calling surgery the science of the knife.

We associate too with category a the way Brent Collins approaches the evolution of his art in respect to orientable, non-orientable, modular and module surfaces, modular spirals and toroids and ribbon sculptures. Spirals are inspired by DNA; the DNA-computing emerging today (see G. Paun, G. Rozenberg, A. Salomaa, Molecular Computation; New Computing Paradigms, Springer, Berlin-New York, 1998) will probably stimulate Brent Collins in his further tentatives."The real reason that I deal with mathematics is that this is what excites me", confesses the sculptor Charles O. Perry, for whom Moebius, the Big Bang, and the broken symmetries of modern physics become terms of reference. For artists of this type, science is not something external to science; science and art emerge concomitantly in mathematics, in physics, biology, music, sculpture, architecture etc.

New ways are opened by Helena Verrill's attempt to approach the problem of finding tessellations that can be folded; many questions that have been answered for Euclidean constructions remain unanswered for origami methods where, for instance, trisection of the angle (impossible in Euclidean geometry) becomes possible. An origami tessellation is for Verrill 
a clear mathematical object: a piecewise linear isometry of the plane which is invariant under some crystallographic group. Four methods of producing origami tessellation are analyzed. The study of patterns grows simultaneously as both art and mathematics.

The most surprising fact in bridging mathematics, art, and physics by the "independent artist" Douglas D. Peden (p. 73-82) is the quantum cosmic connection realized by his "wave space geometry" (recalling the new field of quantum cosmology). As a matter of fact, most contributions in Bridges refer, in some respect or another, to the transgression of the Cartesian creed, of the Galileo-Newtonian paradigm, of classical Aristotelian logic. In Peden's approach (as well as in other contributions in Bridges) the profit is bilateral. Artists enlarge their creative horizon by looking at the achievements of modern science, while scientists have a chance to see, in a new light, their own results.

A deep look at the kernel of the art-science common denominator is realized by Nat Friedman (p. 139-155). A sculpture is defined as an object in a given orientation relative to a fixed horizontal plane. Two sculptures are said to be related if they consist of the same object in different orientations. A hypersculpture (a concept motivated by the work "Attitudes" by Arthur Silverman) is a set of related sculptures. As soon as we perceive the diverse sculptural content of an object, we are motivated to consider it a hypersculpture. For Friedman, the experience of viewing a hypersculpture allows us "to see multiple views from one viewpoint", and this situation leads to a type of what Friedman calls "hyperseeing", meaning seeing in a hyperspace (i.e., a four-dimensional space). The philosophy behind this approach is the simple, but deep, idea that we understand objects in a $\mathrm{n}$-dimensional space by looking at them from a $(n+1)$ dimensional space. We experience this truth when $\mathrm{n}$ is equal to 2 . But sculptures are threedimensional objects, so we need to look at them from a 4-dimensional space; in the absence of it, we try to approximate it with means available in the 3-dimensional space. Reference to Henry Moore and Max Bill illustrate the author's slogan: "To hypersee a sculpture means to visualize it completely from all-around". The objects called "knots" in topology provide an ideal source of hypereseeing and hypersculptures, because "they look quite different when viewed from different directions". Friedman convinces us that knot theory and hypersculptures are interacting with profit for both parts. One remark, however, deserves to be made. In English, more than in other languages, "to see" means also "to understand". Which of them is considered by Friedman? Following Rene Thom, what we see is continuous, while what we understand is finite, i.e., discrete. It follows that (to see, to understand) is a conjugate pair; better we see, worse we understand. Seeing is improved at the expense of understanding and conversely. A second remark concerns the link between Friedman's approach to hyperseeing and the role of higher dimensions in physics and in geometry. With the emergence of non-Euclidean geometries, it became a fashion to refer to a metaphorical four-dimensional space in literature and art (see the book by Henderson, quoted by Friedman). On the other hand, the Big Bang scenario refers to a hypothetical initial ten-dimensional space, further separated in two spaces, one of dimension 4 , the space-time of relativity. The attempts to approximate the ten-dimensional space, where only one physical force was active by means of our 3-dimensional space, is obviously much more difficult, but of the same type as Friedman's problem. John Sharp's pleading for science (especially mathematics) as part of art restoration (p. 157-165) should be known by any student in the field, in order to avoid errors. Delicate problems related to anamorphosis of the skull are 
discussed in relation with Holbein's "The Ambassadors", and it is convincingly argued that mathematics and computers cannot be avoided in this respect (our category a). Sharp does not limit his considerations to technical aspects; he adopts a cultural-historical horizon and points out several errors due, to a large extent, to insufficient cultural and historical information. The same author, in another contribution (p. 275-276), gives a telegraphic message about sliceform sculptures to which he dedicated a book in 1995. George W. Hart (p. 195-202) finds, in the chiral icosahedral symmetry group, a source of creativity in sculpture. Adopting the same large cultural-historical horizon as Sharp, Hart argues with success for the genuine art located in geometry and, at the same time, for the rich science located in art (in this case, sculpture). As a matter of fact, for Hart geometry is married to algebra, due to the essential involvement of a group. Pushing comments far away in the past, starting with Plato, Pythagoras and Da Vinci and giving motivation for all historical steps, Hart proves to be not only an artist and a scholar, but also an excellent teacher. Another good scholar and teacher is Don R. Schol, in his pleading for the integration of mathematics in 3-dimensional design (p. 203-207). Starting from Euclid's Elements, Schol arrives at the need of what he calls "spatial exercises" (exploration of the third dimension) and then to the transformation from the illusionary 2-dimensional world of Euclidean shapes to the reality of the 3-dimensional world. Schol reaches in this way the world of fractals, to which he pays much attention. Since fractals occur in many contributions to Bridges, some clarifications are necessary, in respect to the accent put on their fractional dimension. It is true that in his initial definition of fractals, Mandelbrot makes from the dimensional aspect the main criterion of a fractal, by requiring the Hausdorff-Besicovitch dimension not to be an integer. But at the end of his 1983 book (p. 361) he reconsiders this problem, throwing a doubt on the importance of the dimensional aspect of a fractal. In the book edited by Peitgen and Saupe (p. 25-26), the dimension disappears from the definition of fractals and, what is more important, in Mandelbrot's more recent book, Fractales, hasard et finances, Flammarion, Paris, 1997, the main criteria in recognizing a fractal are the scaling principle, the self-similarity and the invariance with respect to some transformations. The dimension does not disappear, but it is no longer a definitional criterion. As a matter of fact, important fractals, such as the trace of the Brownian motion, have their dimension equal to an integer. In a more careful examination, we realize that there is no sharp distinction between fractal and non-fractal, there are only degrees of fractalness. This remains a topic for further investigation (for instance, the Fibonacci sequences may acquire a status of semi-fractalness, as well as some processes occurring in Escher's work and in Hofstadter's Gödel, Escher, Bach). The theoretical status of fractalness is not yet elucidated. Martin Golubitsky and Ian Melbourne, as mathematicians, answer a question raised by the art historian George Hersey: Could we use the ideas of symmetry breaking to help classify the architectural columns? In order to obtain the answer (under the form of 29 symmetry classes of columns), Hersey's question is transformed in a purely mathematical problem, by assimilating a column with a diformed cylinder and by viewing column symmetries as the subgroup of the symmetries of the cylinder that preserve the column. The main criterion in the classification is related to the nature of the symmetries of the column. Are they continuous, discrete and infinite, or finite? It would be interesting to compare this classification with other classifications of columns, for instance those due to Umberto Eco and Gabriela Ghioca (published in the seventies in Semiotica). Ben Nicholson, Jay Kappraff and Saori Hisano 
(the second a mathematician, the others architects) propose "a system of geometry which may have led to many of the ancient masterpieces of architecture and design". This is a very elaborated work, leading to the conjecture (or conclusion?) that "the Laurentian pavement is a near complete taxonomy of ancient geometry". It belongs to the field of "sacred geometry", to which a systematic attention was paid some decades ago by the journal Scripta Mathematica. The tentative of the authors recall - "toute proportion gardee" - the indo-european reconstruction operated in linguistics. Thomas Michael Stephens confesses two interesting personal experiences in creativity and in art teaching (p. 280-281 and p. 289-290), "I came to mathematics as I did to science, theatre, and art; for the visualizable demonstration of such literary mythos through number, geometry, topology - the shape of things to come". Sorry, he did not develop more. Carol Martin Watts and Donald J. Watts give a telegraphic message on some traces of the geometrical ordering of Roman Florence (p. 281), while the artist Clifford Singer (p. 283-286) gives us an idea of his "geometrical poetry defined in pictorial space". Steve Padget is involved in the way Christopher Wren was dedicated to the restored building of the new St. Paul's Cathedral; Padget concludes that "Wren, like Kepler before him, was attempting the marriage of the symbolic with the empirical and the esoteric with the exoteric. The stone of the cathedral is its visible medium of expression. The geometry is its invisible meaning".

\section{Music}

David Gerhard's (p. 37-47) point of departure is the premise that relative pitch perception (i.e., the identification of the relationship between two successive pitches without identifying the pitches themselves) is better than absolute pitch perception (i.e., the identification of the pitch of a single note, without relating it to another note). In other words, the task of interval detection is easier than the task of absolute pitch detection. On this basis, Gerhard approaches the former task, in contrast with most pitch algorithms, whose aim is to fulfill the latter task. Pozzi Escot (p. 69-72) gives a general account on "the poetics of mathematics in music". Three models (all involving mathematics) are proposed for their explanatory capacity: a model based on the graphic notation of Hildegard von Bingen's antiphon "Sed diabolus", where the phrases' geometry is drawn taking four vertices (onset, apex, nadir, and decay pitches) as definition of its contour; a second model, taking all the Golden Mean parameters of the North American Kwakuitl "Raven Song"; a third model, of the composition "Duet" by the American composer Milton Babbitt. Such models guide the musical creativity; they "do not guarantee the eternal perfection of the composition, they just help us with another comprehension of the work. What the mathematical models provide is a world of common denominators for all music from Africa, Europe, and the Americas". In contrast with the common belief that in the period of the classics and romantics in Europe in the XVIIth and the XVIIIth centuries (exception of J. S. Bach), mathematics was absent from the activity of composers. Escot claims that Franz Schubert and Frederic Chopin, composers of the XVIIIth century, arranged their music with "wondrous orders". Escot helps us to understand the common denominator of music and visual art; it includes the Golden Mean and the fractals as well as various kinds of symmetries. The artistic relevance of the Golden Mean cannot be separated from its remarkable mathematical status: its development in a continuous fraction is the simplest possible, because it uses only the digit 1 , 
iterated infinitely many times. This is what happens with the whole mathematics underlying art; it has an aesthetic status on its own, before (or because?) having aesthetic relevance for music or visual arts. Analyzing the mathematics of Steve Reich's "Clapping Music" (based on rhythm alone), consisting of eight claps and four pauses, arranged in a pattern of three claps, followed in turn by a pause, two claps, a pause, one clap, a pause, two claps, and a pause. That could be performed by musicians without instruments, other than their bodies. Joel K. Haack (p. 87-92) identifies a cyclic permutation acting on the contents of the beats of the original pattern. But Haack does not stop here; he reaches a partial answer to the question of "Why Clapping Music?"; then, other questions come: how many compositions could be created from patterns of eight claps and four non-adjacent rests? What compositions alternative to the structure of "Clapping Music" can be obtained, etc.? Ultimately, Haack provides "an auditory realization of such mathematical ideas as the action of a group element, the computation of a least common multiple, and the proposition from group theory that the order of the product of two disjoint cycles is the least common multiple of the lengths of the cycles". This is the general itinerary of mathematical connections in art: when we identify a mathematical pattern hidden in a work of art, we identify, in a next step, a larger artistic potential of the respective pattern. Haack splendidly confirms this law.

Let us observe that the action of a group element was also used by Dan Vuza (see his articles in the eighties and nineties in Journal of Musical Theory) in respect to Anatol Vieru's modal music. John V.C. Nye (p. 129-138) is concerned with the delicate problem of the relation between subjectivity and measurement in audio reproduction. It is remarkable how Nye succeeds to transform an apparently purely technological issue - the audio reproduction - to a basic cultural dilemma: "The conflict between measurement-oriented professionals and the most anti-scientific audiophiles (some of whom reject all attempts of measurement) would seem to be a perfect case of C.P. Snow's two cultures colliding. But this ignores the large number of scientifically trained professionals who are in the same camp as the hobbyst and performing artist. The conflict arises from too narrow an interpretation of the demands of scientific methodology and an unreasonable fear of judging sound and reproduction on the basis of perceptions that may turn out to be illusory". Nye points out the difficulty of summarizing a multi-dimensional phenomenon with a one-dimensional measure, the so-called problem of aggregation. Indeed, this problem has been proved to lead to genuine difficulties. We have in view two impossibility theorems, one due to Kenneth Arrow (Social Choice and Individual Values, Wiley, New York, 1963), and the other due to Gheorghe Paun (published in Fuzzy Sets and Systems, 9 (1983), p. 205-210). Both these theorems point out the impossibility of reaching a reasonable aggregation of different indicators in a unique one. Jarmila Doubravova (p. 277278) is concerned with Knobloch's "interpersonal hypothesis" claiming that the independent listeners may be able to distinguish interpersonal tendencies in the musical compositions from various periods to the degree that is beyond coincidence". Arun K. Mitra (p. 282) points out that one can obtain the equation of the surface of a violin plate and aims to produce its exact form, to add the appropriate thickness and to study the vibrational properties of free violin plates. Daniel Fitzgerald (p. 291) claims that if a cyclic group is used to model a twelve-tone scale, it turns out that a diminished chord is a subgroup of order four and from that certain properties follow. 


\section{Mathematics}

Jorge Carrera Bolanos (p. 83-86) attempts to identify the basic ideas of chaos theory in the story of the fall of the Aztec Empire almost five hundred years ago. However, attractors, vector field, and chaos become so vague in Balanos' interpretation, that we are left with a feeling of frustration. Other statements need to be supplemented with some remarks. The importance of mathematics as a metaphor of the world is unquestionable, but this is possible because mathematics develops metaphorical processes in its own internal life. It is true that mathematics is not dominated by experiment to the extent to which chemistry is, but it is also true that in the last decades, under the influence of the information-computation revolution, the experimental component of mathematics increased in importance (for instance, what we call a mathematical proof is no longer a purely logical-deductive activity, it may have an empirical-experimental component, when computers are used in some parts of the demonstrative process. On the other hand, new fields of mathematics emerge, such as experimental geometry and experimental number theory).

One of the most comprehensive contributions to Bridges belongs to Reza Sarhangi (a mathematician) and Bruce D. Martin (a chemist) and concerns the circle as a cultural symbol. The presence of circle in various religions and traditions, in science and in art, is so rich that no presentation could exhaust it. Let us recall that it is one of the four fundamental symbols, the other three being the center, the cross and the square. But the center belongs to the definition of the circle, the passage from square to circle, for instance in "mandala", is that of crystalization in "nirvana", while the circle inscribed in a square is a well known symbol of kabbalists. The cross is represented by two ortogonal diameters of the circle, so all fundamental symbols are together. Number pi, DaVinci's man in a circle and a square, axiomatics of geometrey from Euclid to Hilbert, non-Euclidean geometries, Klein's and Poincare's models of hyperbolic geometries, Escher's works, heaven and earth in terms of circles, the kissing numbers, the golden section in a circle, Rumi's poetry, circles of Hell in Dante's "Inferno", Goethe's "Faust", the use of circles in a Grimm brothers' tale, Locke's philosophy, Walt Whitmann's "Leaves of Grass", Black Elk's reflections and Edwin Markham's poetry are only a part of the examples brought to attention by the authors in their pleading for the importance of the circle. If we try to imagine a possible continuation of this research, we could propose more concentration on the aspects related to the word "paradox" occurring in the title. This requires us to consider the circularity as a universal paradigm (in the sense of Thomas Kuhn). Its emergence began with Copernicus, but the very dominance of circularity belongs to the xxth century, due to the increasing importance of the paradoxical, circular situations in art, science and social life. From vicious circles, as they were pointed out as pathological phenomena, by Cantor, Russell, Berry, Grelling and many others, we moved step by step to a status of normality and centrality of the paradox (conceived mainly as the transgression of the principle of non-contradiction). Bohr and Heisenberg in physics, Gödel in mathematics, Maturana and Varela in biology are only some of the scholars who determined this shift towards a rehabilitation of the vicious circles. Typical in this respect are the books by Jon Barwise and Lawrence Moss, Vicious Circles, Stanford, CSLI Publications, 1996, and Jon Barwise and John Etchemendy, The Liar: an Essay in Truth and Circular, Oxford Univ. Press, 
1987. The subject-object circularity is increasing now in importance, while in computer science and in non-classical logics circularity is essential. Let us observe that Sarhangi-Martin's contribution is one of the few in Bridges paying much attention to poetry.

Stephen Eberhart (p. 121-127) proposes some "Pythagorean and Platonic bridges between geometry and algebra", that may have an important impact in building bridges between what students learn in different disciplines. Etymology, art history, philosophy, geometry, algebra, arithmetics are articulated in surprising links, where geometry is the eye of the right hemisphere, while algebra is the eye of the left hemisphere. Mara Alagic (p. 237-244) offers a purely technical investigation concerning "a visual presentation of rank-ordered sets". The motivation comes from theoretical computer science.

\section{Semiotics}

It is well known how important the passage (requiring several centuries) from Roman numerals to Arabic numerals was for Europe. Arithmetic operations became much easier. In 1902, Charles S. Peirce, the founding father of Semiotics, approached a similar problem for (mathematical) logic. He devised a notation that is like the logic alphabet, by introducing an iconic notation for the sixteen binary connectives. Like many other writings of Peirce, this manuscript remained unpublished and perhaps it is still unpublished, because the series of volumes, Writings of Charles S. Peirce, A Chronological Edition (Indiana University Press) did not yet reach the year 1902. Ten years before seeing Peirce's manuscript, Shea Zellweger devised a logic alphabet (see Semiotica, vol. 38, 1985, p .17-54), while in 1997 Glen Clark and Shea Zellweger published new contributions in relation to Peirce's iconic notation. Their joint paper in Bridges (p. 113-119) continues this research. The mirror logic they propose successfully compements Peirce's approach. Ross McCluney (p. 167-174) approaches a very interesting semiotic problem: How can we give a meaning to very large numbers, almost never used in our everyday life and for which human intuition no longer operates? This is a part of a more general problem, fundamental in the XXth century, when science became more and more concerned with phenomena beyond the macroscopic world. Since the latter is the only one familiar to us, the macroscopic has to account for both the infinitely large and the infinitely small. As a matter of fact, this is a basic general task for both modern art and modern science. For instance, Niels Bohr explained that the limits of the human language are just the limits of our macroscopic universe, where language developed. Then, how can we cope with what is beyond this universe? The only compromise possible is to try to build macroscopic metaphors and models accounting for the infinitely large and the infinitely small. This situation explains the emergence of creative and cognitive models and metaphors in the last decades. McCluney is concerned only with a part of this problem: the very large numbers and the possibility to explain their meaning by means of less large numbers and by various analogies where our senses and our intuition may help. It can be observed that he points out implicitly the iterative nature of sign processes. Signs of very large numbers need in their turn other signs and the process never stops. Solomon Marcus (p. 175-179) shows that the discrepancies between poetry and mathematics can be well understood only if we look at them in the framework of the similarities between poetry and mathematics. Daniel F. Daniel's "Math and Metaphor" (p. 225-236) recalls that about ten years 
ago, at an international congress of mathematicians, a plenary session report had the title "Mathematics as Metaphor". This fact shows the great attention paid today to this problem, yesterday it was almost ignored. There is now a rich literature devoted to metaphor in science, a systematic topic in cognitive science and AI. Some important moments in the study of metaphor in science were the book Models and Metaphors by Max Black (1962) and the collective book edited by A. Ortony, Metaphor and Thought (1979). It happens that we were personally involved in this research (S. Marcus: "The Metaphors of the Mathematical Language", Revue Roumaine de Sciences Sociales, Serie Philosophie-Logique, 14 (1970) 2, 139-145; "The Mathematical Metaphor", Computational Linguistics, Budapest, 9 (1973), 151-161; "Why expressive and suggestive metaphors in the scientific (especially mathematical) language? Revue Roumaine de Linguistique - Cahiers de Linguistique Theorique et Appliquee 27 (1990)1, 25-42; "Metaphor as dictatorship", in J. Bernard, ed., World of Signs-World of Things, OGS, Vienna, 1998, 87-108). Our starting point is the fact that all generalization processes in mathematics are based on creative metaphors (i.e., metaphors concerning not an already existing entity, as in classical rhethorics, but entities that emerge just as a consequence of the metaphorical process). Daniel makes no reference to the bibliography of the mathematical metaphor. Maybe he deliberately did not want to develop such a research, but only to make some personal reflections, under the form of a metaphorical approach to mathematics as metaphor; if so, then he is successful. We like especially his opinion that mathematics "deals with human meanings, and is intelligible only within the context of culture. In other words, mathematics is a humanistic study. It is one of the humanities". As soon as we elude the truth revealed by Daniel, the road towards schizophrenia is open. A bold math-chemistry-Escher bridge is proposed by Bruce $\mathrm{D}$. Martin and Reza Sarhangi (p. 245-253). Their initial statement "Chemical reality is 'somehow' related to mathematical symmetry" is supported with strong arguments. The journal $\mathrm{MATCH}-$ Mathematical Chemistry (Germany) publishes systematically papers supporting this statement. The most original part of this research concerns the ingenious isomorphism pointed out by the authors between organic chemistry's symmetries and the types of symmetry in Escher's tessellations. A typical transdisciplinary problem is investigated by Michael Leyton in his book Symmetry, Causality, Mind (MIT Press); Bridges includes only an abstract (p. 273-274). The problem is that, starting from an existing configuration (or organization), to recover the causal process (history) that produced this configuration. We learn that the 600 page rule-system proposed in this respect was already used in meteorology, radiology, chemical engineering, linguistics, and forensic science. The author tells us that art-works are the maximal memoryobjects that human beings can process. Is this fact related to the status of poetry in respect to Kolmogorov-Chaitin algorithmic information theory? In this perspective, poetry is a case of maximum algorithmic-information complexity.

The book edited by Professor Reza Sarhangi will belong to the fundamental bibliography of art-math interaction.

\section{The reviewer}

Solomon Marcus teaches in the Department of Mathematics of the Romanian Academy, Calea Victoriei 125, Bucherest, Romania. 